\title{
Increased Length of Hospital Stay after Endovascular Abdominal Aortic Aneurysm Repair: Role of Pulmonary Complications
}

\author{
Yang Yang ${ }^{1}$, Erik Lehman ${ }^{2}$, Faisal Aziz ${ }^{3}$
}

1. Vascular Surgery, Drexel University College of Medicine, Philadelphia, USA 2. Surgery, Penn State College of Medicine, Penn State Milton S. Hershey Medical Center, Hershey, USA 3. Cardiac / Thoracic / Vascular Surgery, Penn State College of Medicine, Penn State Milton S. Hershey Medical Center, Hershey, USA

Corresponding author: Yang Yang, yyang0711@gmail.com

\section{Abstract}

\section{Objectives}

The average hospital length of stay plays a significant role in healthcare costs, and is also used as a metric of hospital efficiency. An advantage of endovascular abdominal aortic aneurysm repair (EVAR) is the shorter postoperative time period after the surgery. The purpose of this study is to review the factors associated with increased length of stay after EVAR.

\section{Methods}

The records from American College of Surgeons National Quality Improvement Program (ACS-NSQIP) database in 2013 were obtained using Procedure Participant User File. Pre-, intra-, and post-operative factors were assessed of patients undergoing EVAR in 2013. Multivariable logistic regression analysis was used to identify independent variables for a hospital length of stay of at least seven days.

\section{Results}

A total of 1,991 patients (18.7\% female, 81.3\% males) underwent EVAR in 2013. Among these patients, 223 (11.2\%) had a hospital stay greater than seven days. Variables significantly associated with length of stay in a multivariable model included: total operation time greater than 180 minutes (vs. less than 90 minutes, OR 1.88, CI 1.03-3.41, p = 0.039), postoperative, and intraoperative transfusions (OR 2.60, CI 1.66-4.08, $\mathrm{p}<$ 0.001 ), return to operating room (OR 2.88 , CI 1.55-5.38, $\mathrm{p}<0.001$ ), rupture indication for surgery (OR 5.59 , CI 3.18-9.83, p < 0.001), myocardial infarction (OR 5.85, CI 2.22-15.43, p < 0.001), preoperative transfusion (OR 13.05, CI 4.26-39.99, p < 0.001), and on ventilator greater than 48 hours (OR 49.65, CI 10.72-230.07, $\mathrm{p}<$ $0.001)$.

\section{Conclusions}

Received 01/24/2019

Review began 02/22/2019 Review ended 06/17/2019 Published 06/24/2019

\section{(c) Copyright 2019}

Yang et al. This is an open access article distributed under the terms of the Creative Commons Attribution License CC-BY 3.0., which permits unrestricted use, distribution, and reproduction in any medium, provided the original author and source are credited.
Multiple factors affect length of hospital stay in patients who have undergone EVAR. Patients with postoperative respiratory failure after EVAR have a significantly higher risk for longer hospital stays.

Categories: Cardiac/Thoracic/Vascular Surgery

Keywords: evar, respiratory failure, length of stay, aortic aneurysm

\section{Introduction}

In the United States health care system, there has been an emphasis on providing the best patient care while simultaneously maximizing hospital efficiency and expenditures. One factor that is commonly analyzed is length of stay (LOS), which is used as a proxy for resource utilization and healthcare delivery cost [1-3]. Previous studies have shown that decreased LOS results in significant cost savings [3-5]. By understanding factors that play a role in LOS, hospital administration and leaders may be able to improve unnecessarily long hospital stays without negatively impacting patient outcomes.

Abdominal aortic aneurysms (AAA) with large diameter aneurysms are known to have an increased risk of rupture, and in turn, mortality. This disease process can be treated with either open AAA repair or endovascular abdominal aortic aneurysm repair (EVAR). EVAR is quickly becoming the preferred treatment for patients with AAA, due to its shorter postoperative LOS compared to open repair.

The aim of our study was to examine predictors for a prolonged LOS in patients who have undergone EVAR in order to identify potential modifiable risk factors and areas to improve healthcare delivery. We hypothesize that a number of preoperative, intraoperative, and postoperative variables could be identified and used as targets to reduce LOS in patients undergoing EVAR. 


\section{Materials And Methods \\ Data set}

The American College of Surgeons-National Surgical Quality Improvement Program (ACS-NSQIP) is a national outcomes-based, surgical data set comprised of data from over 600 hospitals across the US [6]. It does not identify hospitals, healthcare providers, or patients, thus obviating the need for Institutional Review Board approval or patients' consent. Patient demographics, pre-operative, intra-operative, and postoperative variables are recorded and maintained by trained clinical nurses at all the participating sites. A systemic sampling method is used to ensure adequate representation of all surgical operations. Outcomes are recorded for 30 days and have been shown to be highly reliable with less than $1.5 \%$ variable disagreements in annual audits [7]. The data set is maintained by the ACS and is compliant with the Health Insurance Portability and Accountability Act (HIPAA). Multiple publications based on analyses of this database have been published in literature.

\section{Patients}

ACS started using specific Procedure Targeted Participant User Files in year 2011 for certain vascular surgery and colorectal surgical operations. The procedure targeted file for patients who underwent EVAR in year 2013 was utilized. Using unique case identification numbers, this file was merged to the main ACS-NSQIP adult participant use data file for year 2013. Methods used to extract data from this data set have been described in previously published literature [7-11].

\section{Outcomes}

The primary outcome was increased length of stay, defined as total hospital stay greater than seven days. Basic demographic data was analyzed including age, gender, race, age range, and body mass index (BMI) range. The complete list of pre-operative, intra-operative, and post-operatives is included in Table 1 and Table 2.

\section{Statistical analysis}

All variables were initially summarized with frequencies and percentages or means, medians, and standard deviations. Level of statistical significance was set at $p=0.05$. Logistic regression was used to determine any bivariate associations of independent variables with length of stay greater than seven days. Odds ratios were used to quantify the magnitude and direction of any significant associations. The independent variables that were significant in the initial bivariate analysis were then used in a process of stepwise selection to find the group of variables collectively that were most significantly associated with length of stay greater than seven days in a multivariable logistic regression model. Stringent entry and stay criteria of $p<0.05$ were used for the stepwise process of variable selection to determine the best multivariable logistic regression model that included the factors most significantly associated with increased length of stay. Prior to any modeling selection, the pool of potential predictor variables were tested for multicollinearity using variance inflation factor (VIF) statistics, and those with VIF statistics greater than four were excluded from consideration. Forward, backward, and best subsets methods of variable selection were also employed to check for other potential models, and all four approaches resulted in a similar reduced model. The fit of the final model was assessed using the Pearson, Deviance, and Hosmer and Lemeshow goodness-of-fit tests. Predicted probabilities for patient characteristics were generated from an equation incorporating the parameter estimates from the model. All analyses were performed using SAS software version 9.4 (SAS Institute, Cary, $\mathrm{NC})$.

\section{Results}

\section{Demographics and pre-operative co-morbidities}

A total of 1,991 patients (18.7\% female, 81.3\% males) underwent EVAR in 2013. Among these patients, 223 $(11.2 \%)$ had a hospital stay greater than seven days.

\section{Comparing variables between length of stay greater than seven days and less than seven days}

Patients were divided into two groups: length of stay greater than seven days $(\mathrm{N}=223)$ and length of stay less than seven days $(\mathrm{N}=1,768)$. The following factors were found to be significantly associated with longer length of stay in bivariate analysis using logistic regression: Black race, dyspnea at rest, dependent functional health status, ventilator dependent, severe chronic obstructive pulmonary disease (COPD), congestive heart failure (CHF), open wound or infection, more than $10 \%$ loss of body weight, pre-operative blood transfusion, emergency case, American Society of Anesthesiologists (ASA) classification 4-5: life threatening or moribund, rupture indication for surgery, transferred, access vessels, total operation time greater than 180 , lower extremity revascularization, ischemic colitis, lower extremity ischemia, discharge destination, pneumonia, unplanned intubation, ventilator dependency greater than 48 hours, renal insufficiency, acute renal failure, urinary tract infection, stroke with neurological deficits, cardiac arrest requiring cardiopulmonary resuscitation (CPR), myocardial infarction, perioperative transfusions, deep vein 


\section{Cureus}

thrombosis (DVT), sepsis, septic shock, return to operating room, days from admission to operation greater than one (Table 1 ).

\begin{tabular}{|c|c|c|c|c|c|}
\hline Variable & Total & $\begin{array}{l}\text { Length of stay } \geq 7 \text { days } \\
(N=223)\end{array}$ & $\begin{array}{l}\text { Length of stay }<7 \text { days }(\mathrm{N} \\
=1,768)\end{array}$ & OR (95\% Cl) & $\begin{array}{l}\text { P- } \\
\text { value }\end{array}$ \\
\hline \multicolumn{6}{|l|}{ Preoperative Variables } \\
\hline \multicolumn{6}{|l|}{ Age (years) } \\
\hline$<60$ & $113(5.7)$ & $18(15.9)$ & $95(84.1)$ & Reference & \multirow{4}{*}{0.004} \\
\hline $60-69$ & $\begin{array}{l}487 \\
(24.5)\end{array}$ & $43(8.8)$ & 444 (91.2) & $0.51(0.28-0.93)$ & \\
\hline 70-79 & $\begin{array}{l}796 \\
(40.0)\end{array}$ & $76(9.6)$ & $720(90.4)$ & $0.56(0.32-0.97)$ & \\
\hline$\geq 80$ & $\begin{array}{l}595 \\
(29.9)\end{array}$ & $86(14.5)$ & $509(85.5)$ & $0.89(0.51-1.55)$ & \\
\hline \multicolumn{6}{|l|}{ Sex (gender) } \\
\hline Female & $\begin{array}{l}373 \\
(18.7)\end{array}$ & $55(14.8)$ & 318 (85.2) & $1.49(1.08-2.07)$ & \multirow{2}{*}{0.017} \\
\hline Male & $\begin{array}{l}1618 \\
(81.3)\end{array}$ & $168(10.4)$ & $1450(89.6)$ & Reference & \\
\hline \multicolumn{6}{|l|}{ Race } \\
\hline Non-Hispanic White & $\begin{array}{l}18.24 \\
(91.6)\end{array}$ & 191 (10.5) & 1633 (89.5) & Reference & \multirow{3}{*}{0.003} \\
\hline Non-Hispanic Black & $121(6.1)$ & $24(19.8)$ & $97(80.2)$ & $2.12(1.32-3.39)$ & \\
\hline Hispanic & $46(2.3)$ & $8(17.4)$ & 38 (82.6) & 1.80 (0.83-3.92) & \\
\hline \multicolumn{6}{|l|}{ BMI } \\
\hline <25 (normal) & $\begin{array}{l}515 \\
(26.3)\end{array}$ & 80 (15.5) & 435 (84.5) & Reference & \multirow{3}{*}{$<0.001$} \\
\hline 25-30 (overweight) & $\begin{array}{l}769 \\
(39.3)\end{array}$ & $68(8.8)$ & 701 (91.2) & $0.53(0.37-0.75)$ & \\
\hline$\geq 30$ (obese) & $\begin{array}{l}673 \\
(34.4)\end{array}$ & $65(9.7)$ & 608 (90.3) & $0.58(0.41-0.83)$ & \\
\hline \multicolumn{6}{|l|}{ Inpatient/outpatient } \\
\hline Inpatient & $\begin{array}{l}1976 \\
(99.2)\end{array}$ & 223 (11.3) & $1753(88.7)$ & Reference & \multirow{2}{*}{0.980} \\
\hline Outpatient & $15(0.8)$ & $0(0.0)$ & $15(100.0)$ & $\begin{array}{l}<0.001(<0.001- \\
>999.99)\end{array}$ & \\
\hline \multicolumn{6}{|l|}{ Transferred } \\
\hline No & $\begin{array}{l}1790 \\
(89.9)\end{array}$ & $136(7.6)$ & 1654 (92.4) & Reference & \multirow{2}{*}{$<0.001$} \\
\hline Yes & $\begin{array}{l}201 \\
(10.1)\end{array}$ & 87 (43.3) & $114(56.7)$ & $9.28(6.68-12.90)$ & \\
\hline \multicolumn{6}{|c|}{ Days from admission to operation $\geq 1$} \\
\hline No & $\begin{array}{l}1717 \\
(86.2)\end{array}$ & $117(6.8)$ & 1600 (93.2) & Reference & \multirow{2}{*}{$<0.001$} \\
\hline Yes & $\begin{array}{l}274 \\
(13.8)\end{array}$ & $106(38.7)$ & $168(61.3)$ & $8.63(6.35-11.73)$ & \\
\hline
\end{tabular}




\section{Cureus}

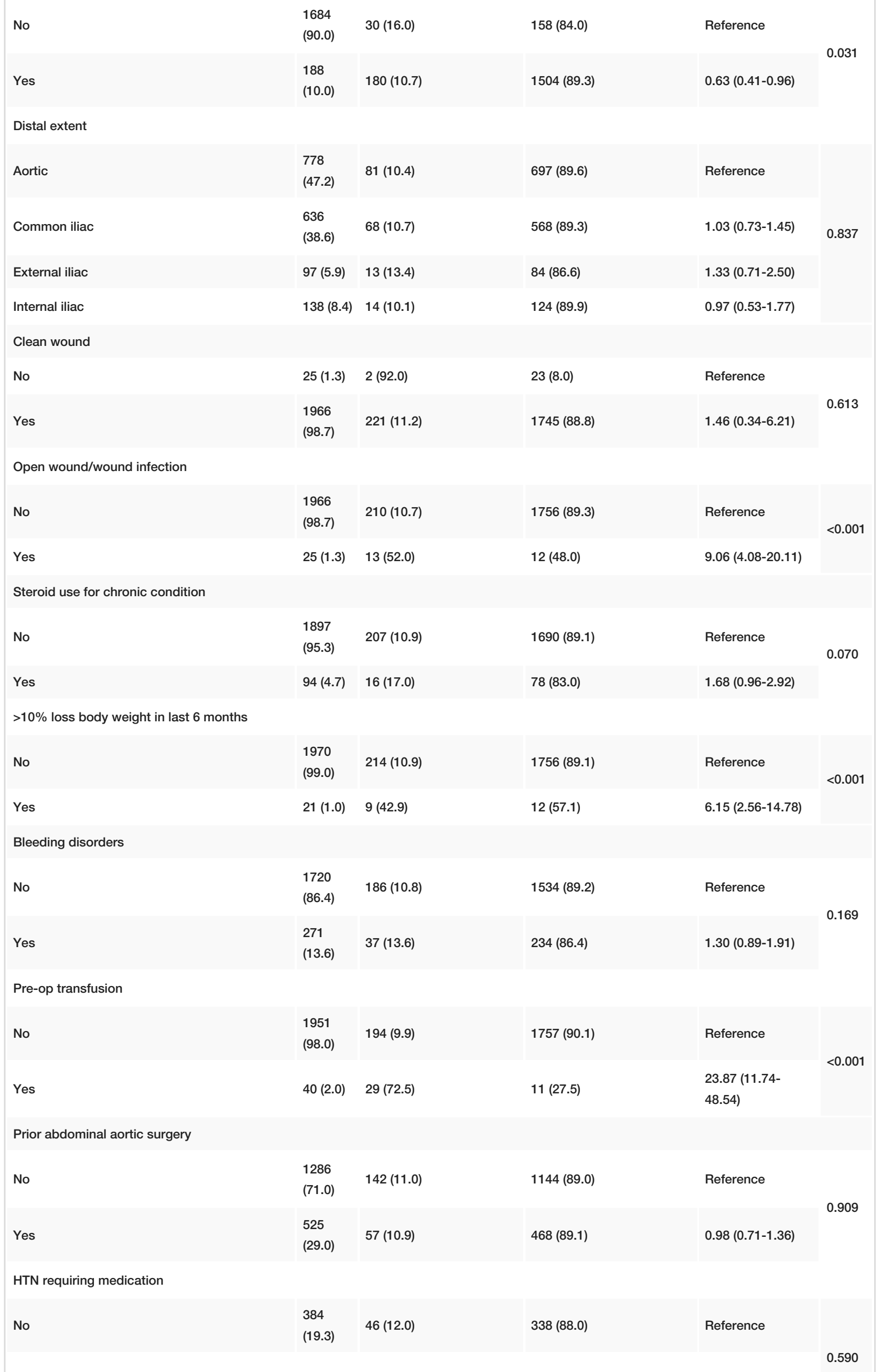




\section{Cureus}

Yes

1607

$177(11.0)$

(80.7)

Diabetes with oral agents or insulin

No

Insulin

Non-insulin

Current smoker within one year

No

Yes

Dyspnea

No

Moderate exertion

At rest

Ventilator dependent

No

Yes

History of severe COPD

No

Yes

Congestive heart failure in $\mathbf{3 0}$ days prior to surgery

No

Yes

Currently on dialysis (pre-operative)

No

Yes

Ischemic colitis

No

No

Yes

Dependent functional health status prior to surgery

No

Yes

Disseminated cancer

1681

(84.4)

$186(11.1)$

69 (3.5) $\quad 9(13.0)$

241

(12.1)

28 (11.6)

1370

$78(12.6)$

(31.2)

8 (12.6)

1577

(79.2)

166 (10.5)

378

$45(11.9)$

36 (1.8) $12(33.3)$

1973

(99.1)

$212(10.7)$

$18(0.9) \quad 11(61.1)$

1638

(82.3)

157 (9.6)

$353 \quad 66(18.7)$

$$
\text { (17.7) }
$$

66 (18.7)

$\begin{array}{ll}1952 & \\ (98.0) & (10.5)\end{array}$

24 (1.2) $5(20.8)$

$\begin{array}{ll}1969 & 209(10.6) \\ (98.9) & \end{array}$

(96.9)

199 (10.4)
213 (88.4)

Reference

$1.21(0.59-2.47)$

0.857

$1225(89.4)$

Reference

$543(87.4)$

$1.21(0.91-1.63)$

1411 (89.5)

Reference

333 (88.1)

$1.15(0.81-1.63)$

4.25 (2.09-8.66)

$24(66.7)$

$<0.001$

$1761(89.3)$

Reference

7 (38.9)

$13.05(5.01-34.03)$

$<0.001$

$1481(90.4)$

Reference

$287(81.3)$

$2.17(1.59-2.97)$

$<0.001$

$1747(89.5)$

Reference

$39(2.0) \quad 18(46.2) \quad 21(53.8)$

$7.31(3.83-13.94)$

$1967 \quad 218(11.1) \quad 1749(88.9)$

19 (79.2)

Reference

0.141

$1760(89.4)$

Reference

8 (36.4)

14.74 (6.11-35.54)

$<0.001$

1716 (89.6)

Reference

$<0.001$

42 (68.9)

$3.90(2.23-6.84)$ 


\section{Cureus}

No

$\begin{array}{lll}1982 & 220(11.1) & 1762(88.9) \\ (99.5) & & \\ 9(0.5) & 3(33.3) & 6(66.7)\end{array}$

Reference

0.051

Yes

$9(0.5) \quad 3(33.3)$

$6(66.7)$

$4.01(1.00-16.13)$

Rupture indication for surgery

No

1819

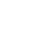

(93.0)

$153(8.4)$

1666 (91.6)

Reference

$137(7.0) \quad 67(48.9)$

$70(51.1)$

$10.42(7.17-15.14)$

Rupture of aneurysm

No

1984

(99.6)

$220(11.1)$

1764 (88.9)

Reference

$7(0.4) \quad 3(42.9)$

$4(57.1)$

$6.01(1.34-27.05)$

Emergency case

No complication

1801

(90.5)

$143(7.9)$

1658 (92.1)

Reference

$190(9.5) \quad 80(42.1)$

$110(57.9)$

$8.43(6.03-11.79)$

Intraoperative Variables

Main body device

Gore Excluder

626

(32.0)

75 (12.0)

$551(88.0)$

Reference

Cook

461

(23.6)

63 (13.7)

398 (86.3)

$0.86(0.60-1.23)$

581

55 (9.5)

$526(90.5)$

$1.30(0.90-1.88)$

Medtronic

(29.7)

$(9.4)$

Other

287

(14.7)

$27(9.4)$

$260(90.6)$

$1.31(0.82-2.08)$

Iliac branched device

No

1708

(85.8)

192 (11.2)

1516 (88.8)

Reference

283

31 (10.9)

$252(0.65-1.45)$

$0.97(0.65-1.45)$

Yes

(14.2)

1946

(97.7)

219 (11.2)

1727 (88.8)

Reference

45 (2.3) $\quad 4$ (8.9)

41 (91.1)

$0.77(0.27-2.17)$

Iliac bare metal stent

No

1922

(96.5) $215(11.2)$

1707 (88.8)

Reference

69 (3.5) $8(11.6)$

$61(88.4)$

$1.04(0.49-2.21)$

General anesthesia

No

$190(9.5) \quad 22(11.6)$

168 (88.4)

Reference

1801

201 (11.2)

1600 (88.8)

$0.96(0.60-1.53)$

0.860

(90.5)

$98(4.9) \quad 2(2.0)$

$96(98.0)$

Reference

1282

80 (6.2)

1202 (93.8)

3.19 (0.77-13.18)

$<0.001$ 


\section{Cureus}

4-5 Life threatening/moribund

609

(30.6)

$468(76.8)$

$14.45(3.52-59.35)$

Vascular surgical specialty

No

Yes

$54(2.7) \quad 3(5.6)$

1937

(97.3)

$220(11.4)$

$51(94.4)$

1717 (88.6)

Reference

$2.18(0.67-7.04)$

EVAR access

Bilateral groin cutdown

Attempted percutaneous access converted to open cutdown

212

(61.1)

$142(11.7)$

$20(1.0) \quad 5(25.0)$

$15(75.0)$

$2.51(0.90-7.02)$

$198 \quad 27(13.6) \quad 171(86.4)$

505 (91.3)

$48(8.7)$

309 (92.0)

$471(94.8)$

$497 \quad 26(5.2)$

687

(34.5)

75 (10.9)

471

(23.7)

95 (20.2)

Acute conversion to open procedure

No

1968

(99.3)

$219(11.1)$

$13(0.7) \quad 4(30.8)$

9 (69.2)

1658 (89.5)

$110(79.7)$

Reference

$2.16(1.39-3.36)$

$138(6.9) \quad 28(20.3)$

1831

(92.0)

$194(10.6)$

$160(8.0) \quad 29(18.1)$

$1637(89.4)$

131 (81.9)

Reference

$1.87(1.22-2.87)$

Hypogastric embolization

No

Yes

Hypogastric revascularization

No

1902

1853

(93.1)

$201(10.8)$

$138(6.9) \quad 22(15.9)$

(95.5)

$210(11.0)$

$89(4.5) \quad 13(14.6)$
1652 (89.2)

$116(84.1)$

$1692(89.0)$

$76(85.4)$
Yes

$1.38(0.75-2.53)$
Reference

$1.56(0.97-2.52)$

Reference

0.298 


\section{Cureus}

No

$\begin{array}{lll}1907 & 201(10.5) & 1706(89.5) \\ (95.8) & & \\ 84(4.2) & 22(26.2) & 62(73.8)\end{array}$

Reference

$3.01(1.81-5.01)$

Postoperative variables

Discharge destination

Home

Expired

Other

Superficial surgical site occurrence

No complication

Superficial incisional SS

Deep incisional SSI

No complication

Deep incisional SSI

Pneumonia

No complication

Pneumonia

Unplanned intubation

No complication

Unplanned intubation

Ventilator $>48$ hours

No complication

On ventilator greater than $48 \mathrm{hrs}$

Urinary tract infection

No complication

Urinary tract infection

Renal insufficiency

No complication

Progressive renal insufficiency

Acute renal failure

No complication

Acute renal failure

CVA/stroke with neurological deficit $\begin{array}{llll}1779 & 108(6.1) & 1671(93.9) & \text { Reference }\end{array}$

$42(2.1) \quad 17(40.5)$

$25(59.5)$

$72(42.6)$

$10.52(5.51-20.08)$

$20.84(14.51$

29.92)

\section{5}

(98.7)

217 (11.0)

1748 (89.0)

Reference

26 (1.3) $6(23.1)$

$20(76.9)$

\section{2}

(99.5)

222 (11.2)

1760 (88.8)

8 (88.9)

$9(0.5) \quad 1(11.1)$

1966

(98.7)

202 (10.3)

25 (1.3) 21 (84.0)

$4(16.0)$

1764 (89.7)

$\begin{array}{ll}1950 & 201(10.3) \\ (97.9) & \end{array}$

1749 (89.7)

19 (46.3)

\section{9}

(97.9)

187 (9.6)

1762 (90.4)

$42(2.1) \quad 36(85.7)$

6 (14.3)

$\begin{array}{lll}1965 & 215(10.9) & 1750(89.1)\end{array}$

$18(69.2)$

$26(1.3) \quad 8(30.8)$

$\begin{array}{lll}1978 & 215(10.9) & 1763(89.1)\end{array}$

5 (38.5)

$13(0.6) \quad 8(61.5)$

1973

(99.1)

209 (10.6)

$18(0.9) \quad 14(77.8)$

4 (22.2)
$2.42(0.96-6.08)$

Reference

$0.99(0.12-7.96)$

Reference

$45.83(15.58$

134.81)

Reference

10.01 (5.36-18.94)

Reference

$56.54(23.51$

135.93)

Reference

$3.62(1.56-8.42)$

Reference

$<0.001$

0.061

0.993

$<0.001$ $<0.001$

$<0.001$ .003

$13.12(4.25-40.46)$

Reference 


\section{Cureus}

\begin{tabular}{|c|c|c|c|c|c|}
\hline No complication & $\begin{array}{l}1982 \\
(99.5)\end{array}$ & $217(11.0)$ & $1765(89.0)$ & Reference & \multirow[t]{2}{*}{$<0.001$} \\
\hline Stroke/CVA & $9(0.5)$ & $6(66.7)$ & $3(33.3)$ & $16.26(4.04-65.46)$ & \\
\hline \multicolumn{6}{|l|}{ Cardiac arrest requiring CPR } \\
\hline No complication & $\begin{array}{l}1975 \\
(99.2)\end{array}$ & $217(11.0)$ & $1758(89.0)$ & Reference & \multirow[t]{2}{*}{0.002} \\
\hline Cardiac arrest requiring CPR & $16(0.8)$ & $6(37.5)$ & $10(62.5)$ & $4.86(1.75-13.51)$ & \\
\hline \multicolumn{6}{|l|}{ Myocardial infarction } \\
\hline No complication & $\begin{array}{l}1960 \\
(98.4)\end{array}$ & $208(10.6)$ & $1752(89.4)$ & Reference & \multirow[t]{2}{*}{$<0.001$} \\
\hline Myocardial infarction & $31(1.6)$ & $15(48.4)$ & $16(51.6)$ & $7.89(3.85-16.20)$ & \\
\hline \multicolumn{6}{|l|}{ DVT/thrombophlebitis } \\
\hline No complication & $\begin{array}{l}1973 \\
(99.1)\end{array}$ & $210(10.6)$ & $1763(89.4)$ & Reference & \multirow{2}{*}{$<0.001$} \\
\hline DVT requiring therapy & $18(0.9)$ & $13(72.2)$ & $5(27.8)$ & $21.82(7.70-61.82)$ & \\
\hline \multicolumn{6}{|l|}{ Sepsis } \\
\hline No complication & $\begin{array}{l}1968 \\
(98.8)\end{array}$ & $208(10.6)$ & $1760(89.4)$ & Reference & \multirow[t]{2}{*}{$<0.001$} \\
\hline Sepsis & $23(1.2)$ & $15(65.2)$ & $8(34.8)$ & $15.87(6.65-37.87)$ & \\
\hline \multicolumn{6}{|l|}{ Septic shock } \\
\hline No complication & $\begin{array}{l}1974 \\
(99.2)\end{array}$ & $210(10.6)$ & $1764(89.4)$ & Reference & \multirow[t]{2}{*}{$<0.001$} \\
\hline Septic shock & $17(0.8)$ & $13(76.5)$ & $4(23.5)$ & $27.30(8.82-84.49)$ & \\
\hline \multicolumn{6}{|l|}{ Lower extremity ischemia } \\
\hline No & $\begin{array}{l}1962 \\
(98.5)\end{array}$ & $215(11.0)$ & $1747(89.0)$ & Reference & \multirow[t]{2}{*}{0.007} \\
\hline Yes & $29(1.5)$ & $8(27.6)$ & $21(72.4)$ & $3.10(1.36-7.08)$ & \\
\hline \multicolumn{6}{|l|}{ Return to OR } \\
\hline No & $\begin{array}{l}1898 \\
(95.3)\end{array}$ & $185(9.8)$ & $1713(90.2)$ & Reference & \multirow[t]{2}{*}{$<0.001$} \\
\hline Yes & $93(4.7)$ & $38(40.9)$ & $55(59.1)$ & $6.40(4.12-9.94)$ & \\
\hline
\end{tabular}

\section{TABLE 1: Bivariate analysis for variables associated with elective surgery.}

BMI: Body mass index; HTN: Hypertension; COPD: Chronic obstructive pulmonary disease; ASA: American Society of Anesthesiologists; EVAR: Endovascular abdominal aortic aneurysm repair; SSI: Surgical site infection; CVA: Cerebrovascular accident; CPR: Cardiopulmonary resuscitation; DVT: Deep vein thrombosis; OR: Operating room.

\section{Multivariable analysis}

The following factors were found to have significant associations with longer hospital stay: total operation time greater than 180 minutes (vs. less than 90 minutes, OR 2.02, CI 1.03-3.41, $\mathrm{p}=0.039$ ), postoperative and intraoperative transfusions (OR 2.60, CI 1.66-4.08, $\mathrm{p}<0.001$ ), return to operating room (OR 2.88, CI 1.55$5.38, \mathrm{p}<0.001$ ), rupture indication for surgery (OR 5.59, CI 3.18-9.83, $\mathrm{p}<0.001$ ), myocardial infarction (OR 5.85, CI 2.22-15.43, $\mathrm{p}<0.001$ ), preoperative transfusion (OR 13.05, CI 4.26-39.99, $\mathrm{p}<0.001$ ), and time on ventilator greater than 48 hours (OR 49.65, CI 10.72-230.07, $\mathrm{p}<0.001$ ) (Table 2). 


\section{Cureus}

\begin{tabular}{|c|c|c|c|}
\hline Variable & OR & $95 \% \mathrm{Cl}$ & P-value \\
\hline Total operation time: $>180$ vs $\leq 90$ & 1.88 & 1.03-3.41 & 0.039 \\
\hline Post-operative/Intra-operative transfusions & 2.60 & $1.66-4.08$ & $<0.001$ \\
\hline Return to OR & 2.88 & $1.55-5.38$ & $<0.001$ \\
\hline Ruptured AAA & 5.59 & 3.18-9.83 & $<0.001$ \\
\hline Myocardial infarction & 5.85 & $2.22-15.43$ & $<0.001$ \\
\hline Pre-operative transfusion & 13.05 & $4.26-39.99$ & $<0.001$ \\
\hline Time on ventilator greater than 48 hours & 49.65 & $10.72-230.07$ & $<0.001$ \\
\hline
\end{tabular}

\section{TABLE 2: Multivariable analysis for variables associated with increased length of stay.}

OR: Operating room; AAA: Abdominal aortic aneurysm.

\section{Predicted probability of increased length of stay}

The probability of an increased length of stay was calculated for all of the factors to be significant in the multivariable analysis. The probability of an increased length of stay was $8.0 \%$ for patients who had operations in the fourth quartile, $10.7 \%$ for patients who required transfusions, $11.8 \%$ for patients who had to return to the operating room, $20.5 \%$ for patients who had rupture as an indication for surgery, $21.3 \%$ for patients who had a myocardial infarction in the post-operative period, $37.6 \%$ for patients who required a pre-operative transfusion, $69.7 \%$ for patients who were on a ventilator for more than 48 hours, and $99.9 \%$ for patients who had all of these seven factors present (Table 3).

\begin{tabular}{|c|c|c|c|c|c|c|c|}
\hline $\begin{array}{l}\text { Operation time } \\
4^{\text {th }} \text { quartile }\end{array}$ & $\begin{array}{l}\text { Post-operative blood } \\
\text { transfusions }\end{array}$ & $\begin{array}{l}\text { Return } \\
\text { to OR }\end{array}$ & $\begin{array}{l}\text { Ruptured } \\
\text { aneurysm }\end{array}$ & $\begin{array}{l}\text { Myocardial } \\
\text { infarction }\end{array}$ & $\begin{array}{l}\text { Pre-operative } \\
\text { transfusion }\end{array}$ & $\begin{array}{l}\text { Ventilator }>48 \\
\text { hours }\end{array}$ & $\begin{array}{l}\text { Probability } \\
(\%)\end{array}$ \\
\hline+ & - & - & - & - & - & - & 8.0 \\
\hline- & + & - & - & - & - & - & 10.7 \\
\hline- & - & + & - & - & - & - & 11.8 \\
\hline - & - & - & + & - & - & - & 20.5 \\
\hline- & - & - & - & + & - & - & 21.3 \\
\hline- & - & - & - & - & + & - & 37.6 \\
\hline - & - & - & - & - & - & + & 69.7 \\
\hline+ & + & + & + & + & + & + & 99.9 \\
\hline
\end{tabular}

TABLE 3: Predicted probability of increased length of hospital stay.

OR: Operating room.

\section{Discussion}

This analysis shows that multiple factors play a role in LOS greater than seven days. Preoperative and intraoperative variables include preoperative transfusion, rupture as an indication for surgery, perioperative transfusions, and greater operation time. Postoperative variables include return to the operating room, myocardial infarction, and ventilator dependency duration greater than 48 hours.

Preoperative variables relating to longer LOS in patients have been extensively studied. In a study done by Siracuse et al., the authors identified dependent functional status as the most significant risk factor for protracted LOS in patients undergoing lower extremity bypass for critical limb ischemia [12]. This was also seen in a study by King et al. evaluating risk factors for increased LOS after elective EVAR. The authors noted the importance of identifying preoperative risk factors such as chronic renal insufficiency, recent weight 
loss, dependent functional status, ASA classification of IV, and dyspnea at rest [13]. These studies emphasize the importance of modifying risk factors in order to optimize surgery but do not evaluate postoperative outcomes as our study has.

Postoperative complication rates have been linked to higher cost of surgical care and have been shown to be more important than preoperative and intraoperative risk factors after major surgery [14]. Additionally, postoperative complications that are more severe can often lead to longer LOS [15]. In our study, postoperative factors that were independently linked to a longer length of stay included return to the operating room, myocardial infarction, and ventilator dependency greater than 48 hours. Patients with postoperative respiratory failure, defined in this study as a failure to wean from a ventilator after 48 hours, had a 50 times greater risk of longer LOS (Table 2), with a predicted probability of almost $70 \%$ (Table 3). Patients who returned to operating room and had postoperative myocardial infarction had a three-fold and six-fold increase in risk for longer hospital stay, respectively. This identifies postoperative respiratory failure as an important risk factor for longer LOS and may present as a target for decreasing healthcare costs and shortening hospital stay. Prior studies have emphasized the significance of respiratory failure in surgery outcomes and have demonstrated its association with mortality and overall cost [14, 16-18].

Length of stay is one of many benchmarks that are used to assess healthcare efficiency as well as cost [1-3]. Given what is known about postoperative respiratory failure and our study results demonstrating its role in patients who have undergone EVAR, optimizing patients' pulmonary status presents as a potential target to improve unnecessarily long LOS and in turn, healthcare costs.

The current study contains some inherent limitations. The NSQIP database is retrospective in nature and therefore has all of the associated limitations of a retrospective study. Outcomes are limited to 30 postoperative days and contain no further follow-up data. Additionally, the database provides no information regarding socioeconomic status or education level. The strength of this study is that NSQIP database has been proven to be reliable and reproducible, and is the most comprehensive surgical database available in the US. Furthermore, the data is collected from multiple institutions throughout the country, thus removing potential geographic biases.

\section{Conclusions}

Multiple factors affect length of hospital stay in patients who have undergone EVAR. Preoperative and intraoperative variables include preoperative transfusion, rupture indication for surgery, perioperative transfusions, and greater operation time. Postoperative variables include return to the operating room, myocardial infarction, and ventilator dependency greater than 48 hours. This study suggests that patients with postoperative respiratory failure after EVAR have a significantly higher risk for longer hospital stays.

\section{Additional Information \\ Disclosures}

Human subjects: All authors have confirmed that this study did not involve human participants or tissue. Animal subjects: All authors have confirmed that this study did not involve animal subjects or tissue. Conflicts of interest: In compliance with the ICMJE uniform disclosure form, all authors declare the following: Payment/services info: All authors have declared that no financial support was received from any organization for the submitted work. Financial relationships: All authors have declared that they have no financial relationships at present or within the previous three years with any organizations that might have an interest in the submitted work. Other relationships: All authors have declared that there are no other relationships or activities that could appear to have influenced the submitted work.

\section{References}

1. Morgan M, Beech R: Variations in lengths of stay and rates of day case surgery: implications for the efficiency of surgical management. J Epidemiol Community Health. 1990, 44:90-105. 10.1136/jech.44.2.90

2. Brasel KJ, Lim HJ, Nirula R, Weigelt JA: Length of stay: an appropriate quality measure? . Arch Surg. 2007, 142:461-466. 10.1001/archsurg.142.5.461

3. Reed T Jr, Veith FJ, Gargiulo NJ 3rd, et al.: System to decrease length of stay for vascular surgery. J Vasc Surg. 2004, 39:395-399. 10.1016/j.jvs.2003.09.015

4. Calligaro KD, Dougherty MJ, Raviola CA, Musser DJ, DeLaurentis DA: Impact of clinical pathways on hospital costs and early outcome after major vascular surgery. J Vasc Surg. 1995, 22:649-660.

5. Mehaffey JH, LaPar DJ, Tracci MC, Cherry KJ, Kerna JA, Upchurch GR Jr: Targets to prevent prolonged length of stay after endovascular aortic repair. J Vasc Surg. 2015, 62:1413-1420. 10.1016/j.jvs.2015.06.219

6. The American College of Surgeons National Surgical Quality Improvement Program . (2019). Accessed: November 11, 2017: https://www.facs.org/quality-programs/acs-nsqip.

7. Shiloach M, Frencher SK Jr, Steeger JE, et al.: Toward robust information: data quality and inter-rater reliability in the American College of Surgeons National Surgical Quality Improvement Program. J Am Coll Surg. 2010, 210:6-16. 10.1016/j.jamcollsurg.2009.09.031

8. Daley J, Khuri SF, Henderson W, et al.: Risk adjustment of the postoperative morbidity rate for the comparative assessment of the quality of surgical care: results of the National Veterans Affairs Surgical Risk Study. J Am Coll Surg. 1997, 185:328-340. 
9. Bilimoria KY, Cohen ME, Ingraham AM, Bentrem D, Richards K, Hall B, Ko C: Effect of postdischarge morbidity and mortality on comparisons of hospital surgical quality. Ann Surg. 2010, 252:183-190. 10.1097/SLA.0b013e3181e4846e

10. Khuri SF, Daley J, Henderson W, et al.: The National Veterans Administration Surgical Risk Study: risk adjustment for the comparative assessment of the quality of surgical care. J Am Coll Surg. 1995, 180:519531.

11. Khuri SF, Daley J, Henderson W, et al.: Risk adjustment of the postoperative mortality rate for the comparative assessment of the quality of surgical care: results of the National Veterans Affairs Surgical Risk Study. J Am Coll Surg. 1997, 185:315-327.

12. Siracuse JJ, Gill HL, Jones DW, et al.: Risk factors for protracted postoperative length of stay after lower extremity bypass for critical limb ischemia. Ann Vasc Surg. 2014, 28:1432-1438. 10.1016/j.avsg.2013.12.027

13. King EG, Farber A, Rybin D, Doros G, Kalish JA, Eslami MH, Siracuse JJ: Preoperative risk factors predict protracted hospital length of stay after elective endovascular abdominal aortic aneurysm repair. Ann Vasc Surg. 2017, 43:73-78. 10.1016/j.avsg.2016.12.017

14. Khuri SF, Henderson WG, DePalma RG, Mosca C, Healey NA, Kumbhani DJ: Determinants of long-term survival after major surgery and the adverse effect of postoperative complications. Ann Surg. 2005, 242:326341. 10.1097/01.sla.0000179621.33268.83

15. McAleese P, Odling-Smee W: The effect of complications on length of stay . Ann Surg. 1994, 220:740-744. 10.1097/00000658-199412000-00006

16. Johnson RG, Arozullah AM, Neumayer L, Henderson WG, Hosokawa P, Khuri SF: Multivariable predictors of postoperative respiratory failure after general and vascular surgery: results from the patient safety in surgery study. J Am Coll Surg. 2007, 204:1188-1198. 10.1016/j.jamcollsurg.2007.02.070

17. Johnson AP, Altmark RE, Weinstein MS, Pitt HA, Yeo CJ, Cowan SW: Predicting the risk of postoperative respiratory failure in elective abdominal and vascular operations using the National Surgical Quality Improvement Program (NSQIP) participant use data file. Ann Surg. 2017, 266:968-974. 10.1097/SLA.0000000000001989

18. Dimick JB, Chen SL, Taheri PA, Henderson WG, Khuri SF, Campbell DA Jr: Hospital costs associated with surgical complications: a report from the private-sector National Surgical Quality Improvement Program. J Am Coll Surg. 2004, 199:531-537. 10.1016/j.jamcollsurg.2004.05.276 